\title{
A NOTE ON THE JACOBIAN CONDITION AND TWO POINTS AT INFINITY
}

\author{
JAMES H. MCKAY AND STUART SUI-SHENG WANG
}

(Communicated by Maurice Auslander)

\begin{abstract}
If two polynomials $F$ and $G$ satisfy the Jacobian condition and the Newton polygon of $F$ has an edge of negative slope, then the sum of terms of $F$ along this edge has at most two distinct irreducible factors and their exponents must be different. Moreover, the slope is either a (negative) integer and the edge touches the $y$-axis or a (negative) Egyptian fraction and the edge touches the $x$-axis. Furthermore, there is an elementary automorphism which reduces the size of the Newton polygon.
\end{abstract}

The main purpose of this note is to prove Theorem 1 and Corollary 4. Part of it was first independently proved by Abhyankar [1, (18.13) Theorem and (18.15) Corollary], Makar-Limanov [4] (which was cited by Vitushkin in [7, p. 416]), and Moh [6, Propositions 4.3 and 4.5], by using different methods. Then it was re-proved by Appelgate and Onishi [1, Lemmas 13, 14, 15, 31 and 33]. The theorem was partially generalized by Charzyński, Chadzyński and Skibiński [3, Theorem 5.1]. Their proofs usually involve many other lemmas. Our proof, as given here, shows that the same elementary ideas which were used in [5] can be used to prove that the Jacobian condition, $\frac{\partial(F, G)}{\partial(x, y)}=1$, implies that $F$ has at most two zeros at infinity. Recall that the Newton polygon for $F(x, y)$ is the convex hull of the origin together with the support of $F$.

Theorem 1. If $F(x, y), G(x, y) \in \mathbb{C}[x, y], \frac{\partial(F, G)}{\partial(x, y)}=1$ and the Newton polygon of $F$ has an edge of negative slope $-\frac{\tilde{n}}{m}$, with $\tilde{n}, \tilde{m}$ as relatively prime positive integers, then this slope is either a (negative) integer or a (negative) Egyptian fraction, neither the right vertex nor the left vertex is on the $45^{\circ}$-line through the origin, and $F_{(\widetilde{n}, \tilde{m})}^{+}(x, y)$, the sum of terms of $F$ along this edge has at most two distinct irreducible factors. Furthermore if $d+1$ is the number of lattice points on the edge (so that $d \geq 1$ ), then exactly one of the following

Received by the editors January 18, 1990.

1980 Mathematics Subject Classification (1985 Revision). Primary 16A72, 12D05; Secondary 13F20, $13 \mathrm{~B} 25$.

Key wora's and phrases. Jacobian condition (Jacobian hypothesis), Newton polygon, points at infinity. 
statements is true:

(a) $\tilde{n}=1, \tilde{m}>1$, the right vertex of the edge is on the $x$-axis, and $F_{(1, \tilde{m})}^{+}(x, y)=\mu x^{l_{1}}\left(x^{\tilde{m}}-\rho y\right)^{d}$ with $\rho \neq 0$ and $0 \leq l_{1} \neq d$.

(b) $\widetilde{m}=1, \widetilde{n}>1$, the left vertex of the edge is on the $y$-axis, and $F_{(\tilde{n}, 1)}^{+}(x, y)=\mu y^{r_{2}}\left(x-\rho y^{\tilde{n}}\right)^{d}$ with $\rho \neq 0$ and $0 \leq r_{2} \neq d$.

(c) $\tilde{n}=\tilde{m}=1$, the edge has the right vertex on the $x$-axis and the left vertex on the $y$-axis, and $F_{(1,1)}^{+}(x, y)=\mu\left(x-\rho_{1} y\right)^{e_{1}}\left(x-\rho_{2} y\right)^{e_{2}}$ with $\rho_{1} \neq \rho_{2}, \rho_{1} \rho_{2} \neq 0, e_{1} \neq e_{2}, e_{1} \geq 0, e_{2} \geq 0$ and $e_{1}+e_{2}=d$.

Proof. Denote the right vertex of the edge by $R\left(r_{1}, r_{2}\right)$ and the left vertex of the edge by $L\left(l_{1}, l_{2}\right)$. Assign weight $(x)=\tilde{n}$, weight $(y)=\tilde{m}$. Then every point on this edge has weight $=l_{1} \tilde{n}+l_{2} \tilde{m}=r_{1} \tilde{n}+r_{2} \widetilde{m}=a$ and

$$
F(x, y)=\sum_{\tilde{i n}+j \tilde{m} \leq a} F_{i, j} x^{i} y^{j}
$$

with $F_{i, j} \in \mathbb{C}$. The sum of terms in $F(x, y)$ along the specified edge is

$$
F_{(\tilde{n}, \tilde{m})}^{+}(x, y)=\sum_{i \tilde{n}+j \tilde{m}=a} F_{i, j} x^{i} y^{j},
$$

and there are at least two distinct non-zero terms in this sum. Let $d+1$ be the number of lattice points on the edge (including the vertices of the edge), then

$$
\begin{aligned}
& r_{1}-l_{1}=d \tilde{m}, \\
& r_{2}-l_{2}=-d \tilde{n} .
\end{aligned}
$$

In terms of this notation,

$$
F_{(\tilde{n}, \tilde{m})}^{+}(x, y)=\mu x^{l_{1}} y^{r_{2}} \prod_{i=1}^{d}\left(x^{\tilde{m}}-\rho_{i} y^{\tilde{n}}\right)
$$

with $\mu, \rho_{i} \in \mathbb{C} \backslash\{0\}$.

Pick a pair of positive integers $u, v$ such that $u \tilde{m}-v \tilde{n}=1$ as in [5, §4]. After the change of variables

$$
\begin{aligned}
& x=U^{u} V^{\tilde{n}}, \\
& y=U^{v} V^{\tilde{m}},
\end{aligned}
$$

$F(x, y)$ and $G(x, y)$ become $A(U, V)$ and $B(U, V)$ respectively. We can write $A(U, V)$ and $B(U, V)$ as Laurent series in $V^{-1}$ with coefficients in $\mathbb{C}(U)$ (so the terms are written in decreasing powers of $V$ ). Note that the weight of any monomial in $x, y$ is exactly the exponent of $V$, after the change 
of variables. Since

$$
\begin{aligned}
A(U, V) & =F\left(U^{u} V^{\tilde{n}}, U^{v} V^{\tilde{m}}\right) \\
& =\sum_{i \tilde{n}+j \tilde{m} \leq a} F_{i, j} U^{i u+j v} V^{\tilde{i n}+j \tilde{m}} \\
& =\alpha(U) V^{a}+\left(\text { terms of } \operatorname{deg}_{V}<a\right),
\end{aligned}
$$

the leading term $\alpha(U) V^{a}$ of $A(U, V)$ is

$$
\begin{aligned}
\alpha(U) V^{a} & =\sum_{i \tilde{n}+j \tilde{m}=a} F_{i, j} U^{i u+j v} V^{i \tilde{n}+j \tilde{m}} \\
& =\mu\left(U^{u} V^{\tilde{n}}\right)^{l_{1}}\left(U^{v} V^{\tilde{m}}\right)^{r_{2}} \prod_{i=1}^{d}\left[\left(U^{u} V^{\tilde{n}}\right)^{\tilde{m}}-\rho_{i}\left(U^{v} V^{\tilde{m}}\right)^{\tilde{n}}\right] \\
& =\mu U^{u l_{1}+v r_{2}+d v \tilde{n}}\left[\prod_{i=1}^{d}\left(U-\rho_{i}\right)\right] V^{\tilde{n} l_{1}+\tilde{m} r_{2}+d \tilde{m} \tilde{n}} \\
& =\mu U^{u l_{1}+v l_{2}}\left[\prod_{i=1}^{d}\left(U-\rho_{i}\right)\right] V^{\tilde{n} l_{1}+\tilde{m} l_{2}} .
\end{aligned}
$$

Thus

$$
\alpha(U)=\mu U^{u l_{1}+v l_{2}} \prod_{i=1}^{d}\left(U-\rho_{i}\right)
$$

and

$$
a=\tilde{n} l_{1}+\tilde{m} l_{2}=\tilde{n} r_{1}+\tilde{m} r_{2}
$$

By the chain rule,

$$
\frac{\partial(A, B)}{\partial(U, V)}=\frac{\partial(F, G)}{\partial(x, y)} \frac{\partial(x, y)}{\partial(U, V)}=U^{u+v-1} V^{\tilde{n}+\tilde{m}-1}
$$

Thus the leading term $\gamma(U) V^{c-1}$ of $\frac{\partial(A, B)}{\partial(U, V)}$, as a Laurent series in $V^{-1}$, is given by

$$
\begin{aligned}
\gamma(U) & =U^{u+v-1}, \\
c & =\tilde{n}+\tilde{m} .
\end{aligned}
$$

According to [5, Theorem 2], there is a $\delta(U) \in \mathbb{C}(U)$ such that

$$
\frac{\gamma(U)}{\delta(U)}=\frac{U^{u+v-1}}{\delta(U)}=\frac{\psi^{\prime}(U)}{\psi(U)}
$$

with

$$
\begin{aligned}
\psi(U) & =\alpha(U)^{c} \delta(U)^{-a} \\
& =\mu^{\tilde{n}+\tilde{m}} U^{\left(u l_{1}+v l_{2}\right)(\tilde{n}+\tilde{m})}\left[\prod_{i=1}^{d}\left(U-\rho_{i}\right)\right]^{\tilde{n}+\tilde{m}} \delta(U)^{-a} .
\end{aligned}
$$


For $\delta(U) \in \mathbb{C}(U)$ and $\theta \in \mathbb{C}$, we let $v_{\theta}(\delta)$ denote the discrete valuation of $\delta$ at the place $\theta$; in other words,

$$
v_{\theta}(\delta)=\text { order of } \delta \text { as : Laurent series in }(U-\theta) \text {. }
$$

Writing $\delta$ as a quotient of two polynomials, $\operatorname{deg}(\delta)$ denotes the degree of the numerator minus the degree of the denominator; in other words,

$$
-\operatorname{deg}(\delta)=\text { order of } \delta \text { as a Laurent series in } U^{-1} \text {. }
$$

Applying [5, Lemma 4] to Equation (*) with $\theta=0$, we have

$$
(u+v-1)-v_{0}(\delta) \text { is } \begin{cases}-1 & \Longleftrightarrow\left(u l_{1}+v l_{2}\right)(\widetilde{n}+\tilde{m})-a v_{0}(\delta) \neq 0, \\ >-1 & \Longleftrightarrow\left(u l_{1}+v l_{2}\right)(\widetilde{n}+\tilde{m})-a v_{0}(\delta)=0\end{cases}
$$

and

(b) $(u+v-1)-\operatorname{deg}(\delta)$ is $\begin{cases}-1 & \Longleftrightarrow\left(u l_{1}+v l_{2}+d\right)(\tilde{n}+\tilde{m})-a \operatorname{deg}(\delta) \neq 0, \\ <-1 & \Longleftrightarrow\left(u l_{1}+v l_{2}+d\right)(\widetilde{n}+\tilde{m})-a \operatorname{deg}(\delta)=0 .\end{cases}$

There are two options for $v_{0}(\delta)$, and two options for $\operatorname{deg}(\delta)$ :

and

$$
v_{0}(\delta)=\left\{\begin{array}{l}
u+v, \\
\frac{\left(u l_{1}+v l_{2}\right)(\tilde{n}+\tilde{m})}{a}=u+v+\frac{l_{1}-l_{2}}{a} ;
\end{array}\right.
$$

There are now four options:

$$
\operatorname{deg}(\delta)=\left\{\begin{array}{l}
u+v, \\
\frac{\left(u l_{1}+v l_{2}+d\right)(\tilde{n}+\tilde{m})}{a}=u+v+\frac{r_{1}-r_{2}}{a} .
\end{array}\right.
$$

$(* *) \quad \operatorname{deg}(\delta)-v_{0}(\delta)=\left\{\begin{array}{l}0, \\ \frac{r_{1}-r_{2}}{a}, \\ \frac{l_{2}-l_{1}}{a}, \\ \frac{\left(r_{1}-l_{1}\right)-\left(r_{2}-l_{2}\right)}{a}=\frac{d(\tilde{n}+\tilde{m})}{a} .\end{array}\right.$

Let $e_{i}$ be the multiplicity of $\rho_{i}$ in $\alpha(U)$. Then

$$
-v_{\rho_{i}}(\delta) \text { is } \begin{cases}-1 & \Longleftrightarrow(\widetilde{n}+\widetilde{m}) e_{i}-a v_{\rho_{i}}(\delta) \neq 0, \\ >-1 & \Longleftrightarrow(\tilde{n}+\tilde{m}) e_{i}-a v_{\rho_{i}}(\delta)=0 .\end{cases}
$$

Either $v_{\rho_{i}}(\delta)=1$ or $1>v_{\rho_{i}}(\delta)=\frac{(\widetilde{n}+\tilde{m}) e_{i}}{a}=$ a positive number; and so $v_{\rho_{i}}(\delta)=$ 1 , i.e., $U-\rho_{i}$ is a factor of $\delta(U)$ of multiplicity 1 . Hence every nonzero root of $\alpha(U)$ is a simple root of $\delta(U)$. Now consider any $\theta \in \mathbb{C}$ with $\theta \neq$ $0, \rho_{1}, \rho_{2}, \ldots, \rho_{d}$, in which case

$$
-v_{\theta}(\delta) \text { is } \begin{cases}-1 & \Longleftrightarrow-a v_{\theta}(\delta) \neq 0, \\ >-1 & \Longleftrightarrow-a v_{\theta}(\delta)=0 .\end{cases}
$$

Thus $v_{\theta}(\delta)=1$ or 0 .

These last two applications of [5, Lemma 4] imply:

(i) $\delta(U)$ is a power of $U$ times a polynomial in $U$, i.e., $\delta(U)$ is a Laurent polynomial;

(ii) any non-zero root of $\delta(U)$ is a simple root; 
(iii) any non-zero root of $\alpha(U)$ is a (simple) root of $\delta(U)$; and

(iv) $\operatorname{deg}(\delta)-v_{0}(\delta) \geq$ (number of distinct roots $\rho_{i}$ 's of $\left.\alpha(U)\right) \geq 1$.

We return to a further consideration of the four options in (**). The fact that $\operatorname{deg}(\delta)-v_{0}(\delta) \geq 1$ rules out the first option immediately.

Case (a). In the case of option (2),

$$
\begin{aligned}
\operatorname{deg}(\delta)-v_{0}(\delta) & =\frac{r_{1}-r_{2}}{a} \\
& =\frac{r_{1}-r_{2}}{r_{1} \widetilde{n}+r_{2} \widetilde{m}} \\
& =\frac{1}{\widetilde{n}}-\frac{r_{2}(\widetilde{n}+\widetilde{m})}{\left(r_{1} \widetilde{n}+r_{2} \widetilde{m}\right) \widetilde{n}} \\
& \leq \frac{1}{\widetilde{n}} .
\end{aligned}
$$

However, $1 \leq$ (number of distinct roots $\rho_{i}$ 's of $\left.\alpha(U)\right) \leq \operatorname{deg}(\delta)-v_{0}(\delta) \leq \frac{1}{n}$. Consequently, $\widetilde{n}=1, r_{2}=0$ and there is only one $\rho_{i}$. Thus a vertex is on the $x$-axis, and

$$
F_{(1, \tilde{m})}^{+}(x, y)=\mu x^{l_{1}}\left(x^{\tilde{m}}-\rho y\right)^{d} .
$$

The option (2) comes from the first option for $v_{0}(\delta)$ in $(\sharp)$ which says that $v_{0}(\delta)=u+v$ and $v_{0}(\delta) \neq u+v+\frac{l_{1}-l_{2}}{a}$. Hence $l_{1} \neq l_{2}=d$.

Case (b). The case of option (3) is similar, with

$$
\begin{aligned}
\frac{l_{2}-l_{1}}{a} & =\frac{l_{2}-l_{1}}{l_{1} \widetilde{n}+l_{2} \widetilde{m}} \\
& =\frac{1}{\widetilde{m}}-\frac{l_{1}(\tilde{n}+\tilde{m})}{\left(l_{1} \widetilde{n}+l_{2} \tilde{m}\right) \tilde{m}} .
\end{aligned}
$$

The conclusion is: $\tilde{m}=1, l_{1}=0$ and there is only one $\rho_{i}$. Thus a vertex is on the $y$-axis, and

$$
F_{(\tilde{n}, 1)}^{+}(x, y)=\mu y^{r_{2}}\left(x-\rho y^{\tilde{n}}\right)^{d} .
$$

The option (3) comes from the first option for $\operatorname{deg}(\delta)$ in (b) which says that $\operatorname{deg}(\delta)=u+v$ and $\operatorname{deg}(\delta) \neq u+v+\frac{r_{1}-r_{2}}{a}$. Hence $r_{2} \neq r_{1}=d$.

Case (c). In the case of option (4), we need to note that this option comes from the combination of

$$
u+v-1-v_{0}(\delta)>-1 \text { and } u+v-1-\operatorname{deg}(\delta)<-1,
$$

i.e.,

$$
u+v-1-v_{0}(\delta) \geq 0 \quad \text { and } \quad u+v-1-\operatorname{deg}(\delta) \leq-2,
$$

which implies that

$$
\operatorname{deg}(\delta)-v_{0}(\delta) \geq 2
$$


However, option (4) is the sum of options (2) and (3) which implies that option (4) requires

$$
\operatorname{deg}(\delta)-v_{0}(\delta) \leq \frac{1}{\widetilde{n}}+\frac{1}{\widetilde{m}} \leq 2
$$

with equality only if $r_{2}=l_{1}=0$, and $\tilde{n}=\widetilde{m}=1$.

Consequently, $\operatorname{deg}(\delta)-v_{0}(\delta)=2$, and $r_{2}=l_{1}=0$. There is a vertex of the edge on each axis; the number of distinct $\rho_{i}$ in $\alpha(U)$ is at most two; and

$$
F_{(1,1)}^{+}(x, y)=\mu\left(x-\rho_{1} y\right)^{e_{1}}\left(x-\rho_{2} y\right)^{e_{2}},
$$

for nonnegative integers $e_{1}, e_{2}$ with $e_{1}+e_{2}=d$. Note that $e_{i}$ is the multiplicity of $\rho_{i}$ in $\alpha(U)$. From $\widetilde{n}=\widetilde{m}=1$ and $l_{1}=r_{2}=0$, we derive that $a=$ $l_{1} \tilde{n}+l_{2} \tilde{m}=l_{1}+l_{2}=l_{2}=d$. We have already shown that $v_{\rho_{i}}(\delta)=1$ for each non-zero root $\rho_{i}$ of $\alpha(U)$. Hence by Equation $(\dagger),(\tilde{n}+\tilde{m}) e_{i}-a v_{\rho_{i}}(\delta) \neq 0$, i.e., $2 e_{i}-d \neq 0$. Thus $e_{1} \neq e_{2}$.

It may help to visualize the three cases of Theorem 1 in terms of edges of negative slope $-\frac{\tilde{n}}{m}$ which are "shallow", i.e., $-1 \leq-\frac{\tilde{n}}{m}<0$, or "steep", i.e., $-\frac{\tilde{n}}{m} \leq-1$.

Corollary 2. Under the hypotheses of Theorem 1, any shallow edge of the Newton polygon must meet the $x$-axis and any steep edge must meet the $y$-axis.

Corollary 3. Under the hypotheses of Theorem 1, exactly one of the following is true:

(a) The Newton polygon of $F$ is either contained within the trapezoid with vertices $(0,0),\left(l_{1}+\widetilde{m} d, 0\right),\left(l_{1}, d\right),(0, d)$ or is a triangle with vertices $(0,0),(\tilde{m} d, 0),(0, d)$.
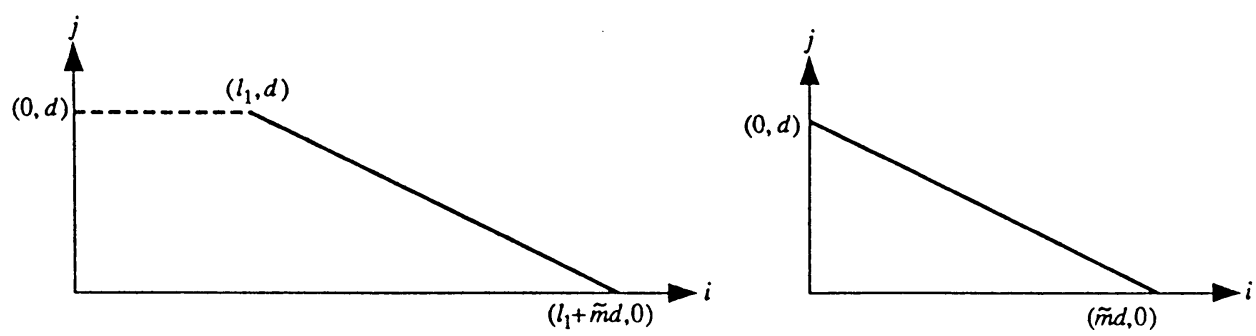

(b) The Newton polygon of $F$ is either contained within the trapezoid with vertices $(0,0),(d, 0),\left(d, r_{2}\right),\left(0, r_{2}+\tilde{n} d\right)$ or is a triangle with vertices $(0,0),(d, 0),(0, \tilde{n} d)$. 

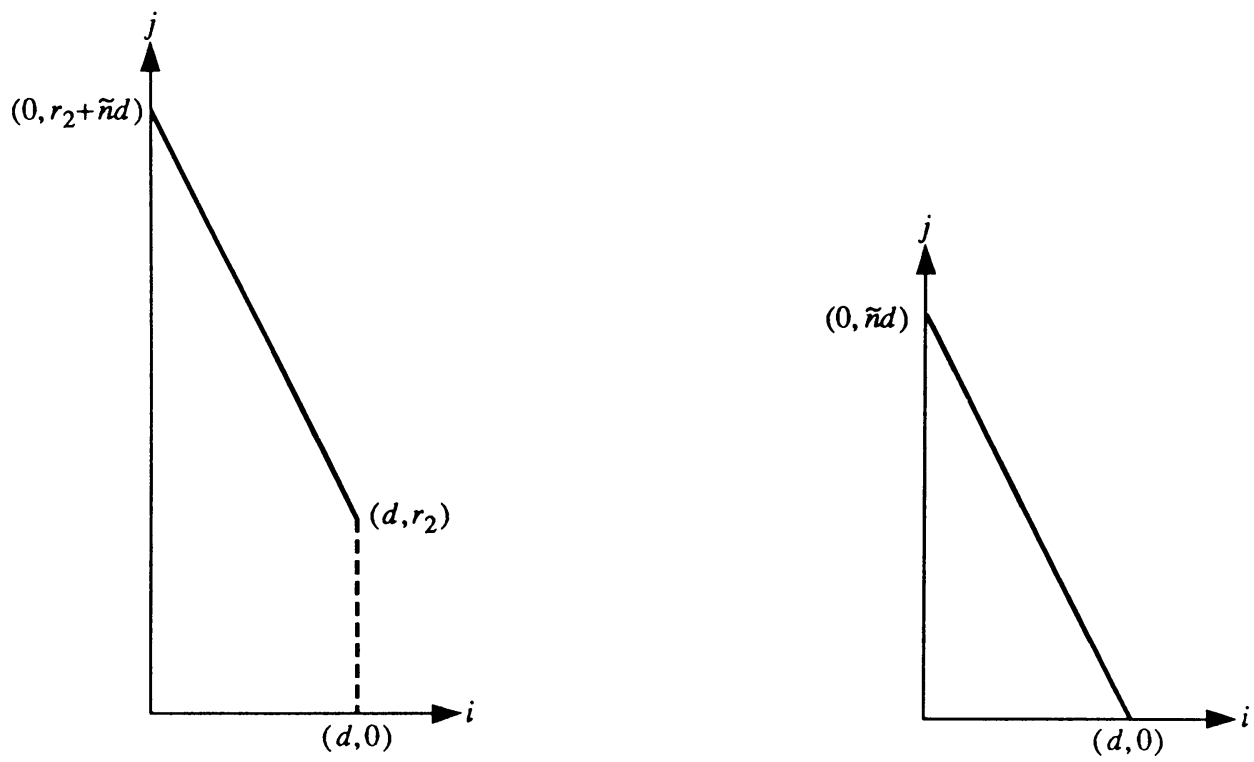

(c) The Newton polygon of $F$ is the isosceles triangle with vertices $(0,0)$, $\left(e_{1}+e_{2}, 0\right),\left(0, e_{1}+e_{2}\right)$.

Proof. Case (a). If $l_{1}=0$, the Newton polygon is a triangle. If $l_{1}>0$ and the Newton polygon is not contained within the stated trapezoid, then the next vertex of the Newton polygon after the vertex $\left(l_{1}, d\right)$ in a counterclockwise direction determines an edge which is contained within the triangle with vertices $(0, d),\left(l_{1}, d\right),\left(0, \frac{l_{1}+\tilde{m} d}{\widetilde{m}}\right)$. This edge is shallow and thus meets the $x$-axis. This is a contradiction because $d \neq 0$.

The case (b) is similar and the case (c) is clear.

Corollary 4. The hypotheses of Theorem 1 imply that there is an elementary automorphism which reduces the area of the Newton polygon for $F(x, y)$.

Proof. Since case (a) and case (b) are similar, we will treat case (b) only.

Case (b). From the statement of Theorem 1,

$$
F_{(\widetilde{n}, 1)}^{+}(x, y)=\mu y^{r_{2}}\left(x-\rho y^{\tilde{n}}\right)^{d}
$$

Consider the elementary automorphism

$$
\begin{aligned}
\phi: \mathbb{C}[x, y] & \rightarrow \mathbb{C}[x, y] \\
x & \mapsto x+\rho y^{\tilde{n}}, \\
y & \mapsto y .
\end{aligned}
$$


We will examine its effect on the Newton polygon. Note that

$$
\begin{aligned}
\phi\left(x^{i} y^{j}\right) & =\left(x+\rho y^{\tilde{n}}\right)^{i} y^{j} \\
& =\sum_{0 \leq k \leq i}\left(\begin{array}{l}
i \\
k
\end{array}\right) \rho^{k} x^{i-k} y^{\tilde{n} k+j} .
\end{aligned}
$$

Under the weight assignment weight $(x)=\tilde{n}$, weight $(y)=1$, every term in the summation has weight

$$
(i-k) \tilde{n}+(\tilde{n} k+j) 1=i \tilde{n}+j 1=\operatorname{weight}\left(x^{i} y^{j}\right) .
$$

This implies that $\phi$. is weight preserving, i.e., points $(i, j)$ in the Newton polygon contribute only to points $(i-k, \tilde{n} k+j)$ on the line of slope $-\frac{\tilde{n}}{1}$ through $(i, j)$. Furthermore, since $0 \leq k \leq i$, the point $(i-k, \tilde{n} k+j)$ is to the left of $(i, j)$ and hence inside the Newton polygon for $F(x, y)$. Thus the Newton polygon for $\phi(F(x, y))$ is contained in the Newton polygon for $F(x, y)$. The automorphism $\phi$ also has the property that $\phi\left(F_{(\widetilde{n}, 1)}^{+}(x, y)\right)=$ $\mu x^{d} y^{r_{2}}$. Therefore the Newton polygon for $\phi(F(x, y))$ does not include the point $\left(0, r_{2}+\tilde{n} d\right)$ which is a vertex of the Newton polygon for $F(x, y)$. Thus the area for the Newton polygon is decreased by this automorphism.

Case (c). From the statement of Theorem 1,

$$
F_{(1,1)}^{+}(x, y)=\mu\left(x-\rho_{1} y\right)^{e_{1}}\left(x-\rho_{2} y\right)^{e_{2}} .
$$

Consider the elementary automorphism

$$
\begin{aligned}
\psi: \mathbb{C}[x, y] & \rightarrow \mathbb{C}[x, y] \\
x & \mapsto \frac{\rho_{2} x-\rho_{1} y}{\rho_{2}-\rho_{1}}, \\
y & \mapsto \frac{x-y}{\rho_{2}-\rho_{1}} .
\end{aligned}
$$

The Newton polygon for $\psi(F(x, y))$ is contained in the Newton polygon for $F(x, y)$. The automorphism $\psi$ also has the property that $\psi\left(F_{(1,1)}^{+}(x, y)\right)=$ $\mu x^{e_{1}} y^{e_{2}}$. Therefore at least one of $\left(e_{1}+e_{2}, 0\right),\left(0, e_{1}+e_{2}\right)$ is not included in the Newton polygon for $\psi(F(x, y))$. Thus the area for the Newton polygon is decreased by this automorphism.

Remark. This still leaves the question of whether or not the Newton polygon for a polynomial $F(x, y)$, which satisfies the Jacobian condition, must have an edge of negative slope.

A similar argument to the proof of Theorem 1 with the change of variables $x=U V$ and $y=V$ establishes

Theorem 5. If $F(x, y), G(x, y) \in \mathbb{C}[x, y], \frac{\partial(F, G)}{\partial(x, y)}=x^{p} y^{q}+$ lower degree terms, then the leading form of $F$ has at most $p+q+2$ distinct irreducible factors. 
The next example shows that the bound $p+q+2$ in Theorem 5 is almost the best possible.

Example 6. Let $F(x, y)=x^{n}-y^{n}$ and $G(x, y)=y$. Then $\frac{\partial(F, G)}{\partial(x, y)}=n x^{n-1}$ so that $p=n-1$ and $q=0$. Now $F$ has $n$ distinct irreducible factors and $p+q+2=n+1$.

\section{REFERENCES}

1. S. S. Abhyankar, Expansion techniques in algebraic geometry, Tata Institute of Fundamental Research, Bombay, 1977, pp. 117-139.

2. H. Appelgate and H. Onishi, The Jacobian conjecture in two variables, J. Pure Appl. Algebra 37 (1985), 215-227.

3. Z. Charzyński, J. Chadzyński and P. Skibiński, A contribution to Keller's Jacobian conjecture, Seminar on deformations (Proceedings, Lódź-Warsaw 1982/84, Lecture Notes in Mathematics 1165), Springer-Verlag, Berlin, Heidelberg, New York, and Tokyo, 1985, pp. 36-51.

4. L. G. Makar-Limanov, 1969, unpublished.

5. J. H. McKay and S. S.-S. Wang, An Elementary proof of the automorphism theorem for the polynomial ring in two variables, J. Pure Appl. Algebra 52 (1988), 91-112.

6. T. T. Moh, On the Jacobian conjecture and the configurations of roots, J. Reine Angew. Math. 340 (1983), 140-212.

7. A. G. Vitushkin, On polynomial transformations of $\mathbb{C}^{n}$, Manifolds-Tokyo 1973 (Proc. Internat. Conf. on Manifolds and Related Topics in Topology, Tokyo, 1973), University of Tokyo Press, Tokyo, 1975, pp. 415-417.

Department of Mathematical Sciences, Oakland University, Rochester Hills, MichiGAN 48309

Department of Mathematical Sciences, Oakland University, Rochester Hills, MichiGAN 48309

Department of Mathematics, Cornell University, Ithaca, New York 14853 